\title{
HÖLDERIAN INVARIANCE PRINCIPLE FOR HILBERTIAN LINEAR PROCESSES *
}

\author{
Alfredas RaČKauskas ${ }^{1,2}$ And Charles Suquet ${ }^{3}$
}

\begin{abstract}
Let $\left(\xi_{n}\right)_{n>1}$ be the polygonal partial sums processes built on the linear processes $X_{n}=$ $\sum_{i \geq 0} a_{i}\left(\epsilon_{n-i}\right), n \geq 1$, where $\left(\epsilon_{i}\right)_{i \in \mathbb{Z}}$ are i.i.d., centered random elements in some separable Hilbert space $\mathbb{H}$ and the $a_{i}$ 's are bounded linear operators $\mathbb{H} \rightarrow \mathbb{H}$, with $\sum_{i>0}\left\|a_{i}\right\|<\infty$. We investigate functional central limit theorem for $\xi_{n}$ in the Hölder spaces $\mathrm{H}_{\rho}^{o}(\mathbb{H})$ of functions $x:[0,1] \rightarrow \mathbb{H}$ such that $\|x(t+h)-x(t)\|=o(\rho(h))$ uniformly in $t$, where $\rho(h)=h^{\alpha} L(1 / h), 0 \leq h \leq 1$ with $0<\alpha \leq 1 / 2$ and $L$ slowly varying at infinity. We obtain the $\mathrm{H}_{\rho}^{o}(\mathbb{H})$ weak convergence of $\xi_{n}$ to some $\mathbb{H}$ valued Brownian motion under the optimal assumption that for any $c>0, t P\left(\left\|\epsilon_{0}\right\|>c t^{1 / 2} \rho(1 / t)\right)=o(1)$ when $t$ tends to infinity, subject to some mild restriction on $L$ in the boundary case $\alpha=1 / 2$. Our result holds in particular with the weight functions $\rho(h)=h^{1 / 2} \ln ^{\beta}(1 / h), \beta>1 / 2$.
\end{abstract}

Résumé. Soit $\left(\xi_{n}\right)_{n \geq 1}$ le processus polygonal de sommes partielles bâti sur le processus linéaire $X_{n}=\sum_{i>0} a_{i}\left(\epsilon_{n-i}\right), n \geq 1$, les $\left(\epsilon_{i}\right)_{i \in \mathbb{Z}}$ étant des éléments aléatoires i.i.d., centrés d'un espace de Hilbert séparable $\mathbb{H}$ et les $a_{i}$ 's des opérateurs linéaires bornés $\mathbb{H} \rightarrow \mathbb{H}$, vérifiant $\sum_{i>0}\left\|a_{i}\right\|<\infty$. Nous étudions le théorème limite central fonctionnel pour $\xi_{n}$ dans les espaces de Hölder $\overline{\mathrm{H}}_{\rho}^{o}(\mathbb{H})$ de fonctions $x:[0,1] \rightarrow \mathbb{H}$ vérifiant $\|x(t+h)-x(t)\|=o(\rho(h))$ uniformément en $t$, où $\rho(h)=h^{\alpha} L(1 / h), 0 \leq h \leq 1$ avec $0<\alpha \leq 1 / 2$ et $L$ à variation lente. Nous prouvons la convergence en loi dans $\mathrm{H}_{\rho}^{o}(\mathbb{H})$ de $\xi_{n}$ vers un mouvement brownien à valeurs dans $\mathbb{H}$, sous la condition optimale que pour tout $c>0$, $t P\left(\left\|\epsilon_{0}\right\|>c t^{1 / 2} \rho(1 / t)\right)=o(1)$ quand $t$ tend vers l'infini, au prix dans le cas limite $\alpha=1 / 2$ d'une légère restriction sur $L$. Notre résultat s'applique en particulier au cas $\rho(h)=h^{1 / 2} \ln ^{\beta}(1 / h), \beta>1 / 2$.

Mathematics Subject Classification. 60F17, 60B12.

Received December 14, 2007. Revised March 11, 2008.

\section{INTRODUCTION}

Let us denote by $\mathrm{C}[0,1]=\mathrm{C}([0,1], \mathbb{R})$ the space of continuous functions $x:[0,1] \rightarrow \mathbb{R}$, endowed with the supremum norm. The classical Donsker-Prohorov invariance principle states the $\mathrm{C}[0,1]$-weak convergence to

Keywords and phrases. Central limit theorem in Banach spaces, Hölder space, functional central limit theorem, linear process, partial sums process.

* Research supported by a French-Lithuanian cooperation agreement "PHC Egide Gilibert".

1 Department of Mathematics and Informatics, Vilnius University, Naugarduko 24, 2006 Vilnius, Lithuania;

alfredas.rackauskas@maf .vu.It

2 Institute of Mathematics and Informatics, Akademijos str. 4, 08663 Vilnius, Lithuania

3 Laboratoire P. Painlevé, UMR 8524 CNRS, Université Lille I, Bât. M2, Cité Scientifique, 59655 Villeneuve d'Ascq Cedex,

France; Charles.Suquet@math.univ-lille1.fr 
some Brownian motion $W$ of the polygonal line $\xi_{n}$ built on the partial sums of a centered, square integrable, i.i.d. sequence $\left(X_{i}\right)_{i \geq 1}$ of real random variables. This result has a lot of applications, especially in statistics, and continues to receive many extensions. Our current contribution involves three directions of extension, dealing with:

- infinite dimensional $X_{i}$ 's;

- other topological frameworks than $\mathrm{C}[0,1]$ for the weak convergence of $\xi_{n}$;

- dependent $X_{i}$ 's.

When the $X_{i}$ 's are i.i.d. random elements in some separable Banach space $(B,\|\|)$, we say that $X_{1}$ satisfies the central limit theorem in $B$, denoted by $X_{1} \in \mathrm{C} L T(B)$, if $n^{-1 / 2} S_{n}:=n^{-1 / 2}\left(X_{1}+\cdots+X_{n}\right)$ converges in distribution in $B$ (the limit is then necessarily some Gaussian random element in $B$ ). It is well-known that the central limit theorem in $B$ is not a direct extension of the finite dimensional case. Depending on the geometry of the space $B$, one can even find some bounded random element $X_{1}$ which does not satisfy the CLT, see $e . g$. [8]. In the nice case where $B$ is a Hilbert space, $X_{1} \in \operatorname{CLT}(B)$ is equivalent to $\mathbf{E} X_{1}=0$ and $\mathbf{E}\left\|X_{1}\right\|^{2}<\infty$. The invariance principle in $B$ inherits the geometric pathologies of the CLT in $B$ in the following sense. Denoting by $\xi_{n}$ the polygonal line with vertices $\left(k / n, S_{k}\right)$, we say that $X_{1} \in \mathrm{FCLT}(B)$ if $n^{-1 / 2} \xi_{n}$ converges in distribution to some $B$-valued Brownian motion in the space $\mathrm{C}([0,1], B)$ of continuous functions $[0,1] \rightarrow B$. In 1973, Kuelbs $[6]$ proved that $X_{1} \in \mathrm{FCLT}(B)$ if and only if $X_{1} \in \mathrm{CLT}(B)$.

Replacing $\mathrm{C}([0,1], \mathbb{R})$ or $\mathrm{C}([0,1], B)$ 's topology by a stronger one, leads to invariance principles of a wider scope than in the classical setting. Indeed this provides more continuous functionals of the paths of $\xi_{n}$. For instance, invariance principles in Hölder spaces have statistical applications to the detection of a changed segment in data $[13,15]$. Let us recall the first Hölderian invariance principle, obtained by Lamperti [7]. For $0<\alpha<1$, let $\mathrm{H}_{\alpha}^{o}[0,1]=\mathrm{H}_{\alpha}^{o}([0,1], \mathbb{R})$ be the vector space of continuous functions $x:[0,1] \rightarrow \mathbb{R}$ such that $\lim _{\delta \rightarrow 0} \omega_{\alpha}(x, \delta)=0$, where

$$
\omega_{\alpha}(x, \delta)=\sup _{\substack{s, t \in[0,1], 0<t-s<\delta}} \frac{|x(t)-x(s)|}{|t-s|^{\alpha}} .
$$

$\mathrm{H}_{\alpha}^{o}[0,1]$ is a separable Banach space when endowed with the norm

$$
\|x\|_{\alpha}:=|x(0)|+\omega_{\alpha}(x, 1)
$$

Lamperti proved that if $0<\alpha<1 / 2$ and $\mathbf{E}\left|X_{1}\right|^{p}<\infty$, where $p>p(\alpha):=1 /(1 / 2-\alpha)$, then $n^{-1 / 2} \xi_{n}$ converges in distribution to $W$ in the space $\mathrm{H}_{\alpha}^{o}[0,1]$. This result was completed and extended by the authors [12] to the case of Banach space valued $X_{i}$ 's in the following way. Put $\rho(h)=h^{\alpha} L(1 / h), 0 \leq h \leq 1$ with $0<\alpha \leq 1 / 2$ and $L$ slowly varying at infinity. Let $\mathrm{H}_{\rho}^{o}(B)=\mathrm{H}_{\rho}^{o}([0,1], B)$ be the Hölder space of functions $x:[0,1] \mapsto B$, such that $\|x(t+h)-x(t)\|=o(\rho(h))$, uniformly in $t$ (the relevant Hölder norm and the technical assumptions on $L$ are explicited below in Section 1). Then $n^{-1 / 2} \xi_{n}$ converges weakly to some $B$-valued Brownian motion in the space $\mathrm{H}_{\rho}^{o}(B)$ if and only if $X_{1} \in \operatorname{CLT}(B)$ and for every positive $c, \lim _{t \rightarrow \infty} t \mathbf{P}\left\{\left\|X_{1}\right\|>c \theta(t)\right\}=0$, where $\theta(t):=t^{1 / 2} \rho(1 / t)$. In the familiar case where $\rho(h)=h^{\alpha}$, the second condition is equivalent to $\mathbf{P}\left\{\left\|X_{1}\right\|>t\right\}=$ $o\left(t^{-p(\alpha)}\right)$.

In view of statistical applications, there is an obvious interest in extending the invariance principles beyond the classical case of i.i.d. observations. A recent survey of invariance principles in $\mathrm{C}[0,1]$ for stationary sequences is [10]. For invariance principles under various weak dependence conditions, let us also mention [3]. Hamadouche [4] gives some Hölderian invariance principles for real valued $\alpha$-mixing or associated $X_{i}$ 's. In a recent contribution, Juodis et al. [5] investigate the invariance principle in $\mathrm{H}_{\alpha}^{o}$ for some linear processes $X_{i}=\sum_{j \geq 0} a_{j} \epsilon_{i-j}$, where $\left(\epsilon_{j}\right)_{j \in \mathbb{Z}}$ are i.i.d., centered and square integrable random variables with $\sum_{j \geq 0} a_{j}^{2}<\infty$. When $\sum_{i>0}\left|a_{i}\right|<\infty$, they show that $n^{-1 / 2} \xi_{n}$ converges weakly in $\mathrm{H}_{\alpha}^{o}[0,1]$ to some Brownian motion under the optimal assumption that $\mathbf{P}\left\{\left|\epsilon_{0}\right| \geq t\right\}=o\left(t^{-p(\alpha)}\right)$.

A natural extension of linear process to infinite dimensional spaces is linear process in the separable Hilbert space $\mathbb{H}$, obtained by replacing the constants $a_{j}$ by continuous linear operators $\mathbb{H} \rightarrow \mathbb{H}$, acting on the i.i.d. 
random elements $\epsilon_{j}$ 's in $\mathbb{H}$ (for a formal definition, see (1.5) below). Merlevède et al. [9] obtained optimal central limit theorem for such processes and this result was completed by Dedecker and Merlevède [2] who established the corresponding functional central limit theorem in $\mathrm{C}([0,1], \mathbb{H})$. The present contribution investigates the functional central limit theorem for such linear processes in the space $\mathrm{H}_{\rho}^{o}(\mathbb{H})$. Our main result extends the short memory case in [5] both with infinite dimensional setting and general Hölderian weights functions $\rho$.

The paper is organized as follows. The main result together with all the preliminary material is presented in Section 1. Proofs are given in Section 2 which starts by a general methodology to prove invariance principles in $\mathrm{H}_{\rho}^{o}(B)$ (Theorem 2.1 which may be of independent interest). Technical lemmas and tools are gathered in Section 3 .

\section{Results}

\subsection{Notations}

Let $(B,\|\|)$ be a separable Banach space. A $B$-valued Brownian motion $W_{Q}$ with covariance operator $Q$ is a Gaussian process indexed by $[0,1]$, with independent increments such that $W_{Q}(t)-W_{Q}(s)$ has the same distribution as $|t-s|^{1 / 2} Y$, where $Y$ is a centered Gaussian random element in $B$ with covariance operator $Q$.

We write $\mathrm{C}(B)$ for the Banach space of continuous functions $x:[0,1] \rightarrow B$ endowed with the supremum norm $\|x\|_{\infty}:=\sup \{\|x(t)\| ; t \in[0,1]\}$. Let $\rho$ be a real valued non decreasing function on $[0,1]$, null and right continuous at 0 , positive on $(0,1]$. Put

$$
\omega_{\rho}(x, \delta):=\sup _{\substack{s, t \in[0,1], 0<t-s<\delta}} \frac{\|x(t)-x(s)\|}{\rho(t-s)} .
$$

We associate to $\rho$ the Hölder space

$$
\mathrm{H}_{\rho}^{o}(B):=\left\{x \in \mathrm{C}(B) ; \lim _{\delta \rightarrow 0} \omega_{\rho}(x, \delta)=0\right\},
$$

equipped with the norm

$$
\|x\|_{\rho}:=\|x(0)\|+\omega_{\rho}(x, 1) .
$$

To discard triviality, we may assume that $\rho(h) \geq c h$ for some positive constant $c$. Then $\mathrm{H}_{\rho}^{o}(B)$ inherits the separability of $B$ (see [11]). As in [12], we shall restrict our study to the case of weight functions $\rho$ in the class $\mathcal{R}$ defined below. For any $\rho$ in $\mathcal{R}$, the space $\mathrm{H}_{\rho}^{\circ}(B)$ supports any $B$-valued Brownian motion.

Definition 1.1. Let $\mathcal{R}$ be the class of non decreasing functions $\rho:[0,1] \rightarrow \mathbb{R}$, positive on $(0,1]$, such that $\rho(0)=0$ and satisfying

i) for some $0<\alpha \leq 1 / 2$, and some positive function $L$ which is normalized slowly varying at infinity,

$$
\rho(h)=h^{\alpha} L(1 / h), \quad 0<h \leq 1 ;
$$

ii) $\theta(t)=t^{1 / 2} \rho(1 / t)$ is $C^{1}$ on $[1, \infty)$;

iii) there is a $\beta>1 / 2$ and some $a>1$, such that $\theta(t) \ln ^{-\beta}(t)$ is non decreasing on $[a, \infty)$.

We say that a function is ultimately decreasing or increasing or non decreasing or non increasing if the corresponding monotonicity holds on some interval $[c, \infty)$.

Remark 1.2. Clearly $L(t) \ln ^{-\beta}(t)$ is normalized slowly varying for any $\beta>0$, so when $\alpha<1 / 2, t^{1 / 2-\alpha} L(t)$ $\ln ^{-\beta}(t)$ is ultimately non decreasing and iii) is automatically satisfied.

The assumption ii) of $C^{1}$ regularity for $\theta$ is not a real restriction, since the function $\rho(1 / t)$ being $\alpha$-regularly varying at infinity (that is $\rho(1 / t)=t^{-\alpha} L(t), \quad t \geq 1$ ) is asymptotically equivalent to a $C^{\infty} \alpha$-regularly varying function $\tilde{\rho}(1 / t)$ (see [1]). Then the corresponding Hölderian norms are equivalent. 
Put $b:=\inf _{t \geq 1} \theta(t)$. Since by iii), the function $\theta(t)$ is ultimately increasing and $\lim _{t \rightarrow \infty} \theta(t)=\infty$, we can define its generalized inverse $\varphi$ on $[b, \infty)$ by

$$
\varphi(u):=\sup \{t \geq 1 ; \theta(t) \leq u\} .
$$

With this definition, we have $\theta(\varphi(u))=u$ for $u \geq b$ and $\varphi(\theta(t))=t$ for $t \geq a$.

The following notation is convenient for the various weak convergences considered in the paper. Let $\mathfrak{X}$ be some separable Banach space and $\left(Y_{n}\right)_{n \geq 1}$ be a sequence of random elements in $\mathfrak{X}$. We write

$$
Y_{n} \underset{n \rightarrow \infty}{\stackrel{\mathfrak{X}}{\longrightarrow}} Y,
$$

for the weak convergence of $\left(Y_{n}\right)_{n \geq 1}$ in the space $\mathfrak{X}$ to the random element $Y$, i.e. $\mathbf{E} f\left(Y_{n}\right)$ converges to $\mathbf{E} f(Y)$ for any continuous and bounded $f: \mathfrak{X} \rightarrow \mathbb{R}$.

For the sequence $\left(X_{n}\right)_{n \geq 1}$ of random elements in the separable Banach space $B$, put

$$
S_{0}:=0, \quad S_{n}:=\sum_{i=1}^{n} X_{i}
$$

and define the partial sums process $\xi_{n}$ by

$$
\xi_{n}(t):=S_{[n t]}+(n t-[n t]) X_{[n t]+1}, \quad t \in[0,1],
$$

where $[n t]$ denotes the integer part of $n t$. As polygonal lines, the paths of $\xi_{n}$ belong to $\mathrm{H}_{\rho}^{o}(B)$ for every $\rho$ in $\mathcal{R}$ since then $\rho(h) \geq c h$ for some constant $c>0$.

In this paper we consider the case where $\left(X_{k}\right)_{k \geq 0}$ is a linear process with values in the separable Hilbert space $\mathbb{H}$ of the form

$$
X_{k}=\sum_{i=0}^{\infty} a_{i}\left(\epsilon_{k-i}\right), \quad k=0,1, \ldots,
$$

where $\left(a_{i}, i \in \mathbb{Z}\right)$ is a given sequence of continuous linear operators $\mathbb{H} \rightarrow \mathbb{H}$ with $a_{i}=0$ for $i<0$ and $\left(\epsilon_{i}, i \in \mathbb{Z}\right)$ is a sequence of independent identically distributed random elements in $\mathbb{H}$ with $\mathbf{E} \epsilon_{0}=0$ and $\mathbf{E}\left\|\epsilon_{0}\right\|^{2}<\infty$. We shall abbreviate the notation $a_{i}\left(\epsilon_{k-i}\right)$ in $a_{i} \epsilon_{k-i}$. In the same spirit, we use the same notation \|\| for the norm in $\mathbb{H}$ and the operator norm on the space of continuous linear operators $\mathbb{H} \rightarrow \mathbb{H}$. If we assume that $\sum_{i \in \mathbb{Z}}\left\|a_{i}\right\|<\infty$ then the series in (1.5) converges almost surely in the strong topology of $\mathbb{H}$ and its sum $X_{k}$ is a random element of $\mathbb{H}$. This follows by Itô-Nisio theorem (see e.g. [8], p. 151), since $\mathbf{E}\left\|\sum_{i} a_{i} \epsilon_{k-i}\right\|^{2} \leq \mathbf{E} \epsilon_{0}^{2} \sum_{i}\left\|a_{i}\right\|^{2}$. Moreover $\left(X_{k}\right)_{k \geq 0}$ is stationary.

\subsection{Main result}

Theorem 1.3. Let $\left(X_{k}\right)_{k \geq 0}$ be the linear process defined by (1.5) and assume that $\left(a_{i}\right)_{i \geq 0}$ satisfies:

$$
\sum_{i=0}^{\infty}\left\|a_{i}\right\|<\infty
$$

Define the continuous linear operator

$$
A:=\sum_{i=0}^{\infty} a_{i}
$$


assume that $A \neq 0$ and denote by $A^{*}$ its adjoint operator. Write $K$ for the covariance operator of the square integrable random element $\epsilon_{0}$ in $\mathbb{H}$. Let $S_{n}$ and $\xi_{n}$ be the partial sums and partial sums process built on $\left(X_{k}\right)_{k \geq 0}$, defined by (1.3) and (1.4). Then for every $\rho \in \mathcal{R}$,

$$
n^{-1 / 2} \xi_{n} \underset{n \rightarrow \infty}{\stackrel{\mathrm{H}_{\rho}^{o}(\mathbb{H})}{\longrightarrow}} W_{Q}
$$

where $W_{Q}$ is a $\mathbb{H}$ valued Brownian motion with covariance operator $Q=A K A^{*}$, if for every $c>0$,

$$
\lim _{t \rightarrow \infty} t \mathbf{P}\left\{\left\|\epsilon_{0}\right\|>c \theta(t)\right\}=0
$$

Condition (1.9) is optimal because the class of linear processes considered includes the special case where $X_{k}=\epsilon_{k}$ and it is known from [12] that in this case (1.9) is necessary for the weak- $\mathrm{H}_{\rho}^{o}(\mathbb{H})$ convergence of $n^{-1 / 2} \xi_{n}$ to $W_{Q}$. It is easily seen that if $\alpha<1 / 2$ in (1.1), then we can drop the requirement "for every $c>0$ " in (1.9) and simply take $c=1$. But this requirement cannot be dropped if $\alpha=1 / 2$, see Remark 12 in [12].

To illustrate Theorem 1.3, it seems worth focusing on the cases $\rho(h)=h^{\alpha}, 0<\alpha<1 / 2$ and $\rho(h)=$ $h^{1 / 2} \ln ^{\beta}(b / h)$ where $\beta>1 / 2$ and $b$ is some positive constant chosen so that $\rho$ increases on $[0,1]$.

Corollary 1.4. If $\rho(h)=h^{\alpha}, 0<\alpha<1 / 2$ in Theorem 1.3, the convergence (1.8) holds if (1.9) is replaced by

$$
\lim _{t \rightarrow \infty} t^{p} \mathbf{P}\left\{\left\|\epsilon_{0}\right\|>t\right\}=0
$$

where $p=(1 / 2-\alpha)^{-1}$.

Corollary 1.5. If $\rho(h)=h^{1 / 2} \ln ^{\beta}(b / h), \beta>1 / 2$ in Theorem 1.3, the convergence (1.8) holds if (1.9) is replaced by

$$
\mathbf{E} \exp \left(d\left\|\epsilon_{0}\right\|^{1 / \beta}\right)<\infty, \quad \text { for each } d>0
$$

\section{Proofs}

\subsection{General reduction}

We describe here a general method to establish the weak- $\mathrm{H}_{\rho}^{o}(B)$ convergence of the partial sums process $v_{n}^{-1} \xi_{n}$ built on random elements $X_{n}$ of the separable Banach space $B$. This may be of independent interest to prove invariance principles under various kind of dependence of the underlying sequence $\left(X_{n}\right)_{n \geq 1}$.

The function $\rho$ is assumed to belong to $\mathcal{R}$ all along the paragraph. According to [14], $v_{n}^{-1} \xi_{n}$ converges weakly to $\xi$ in $\mathrm{H}_{\rho}^{o}(B)$ if and only if

a) the "finite dimensional" distributions of $v_{n}^{-1} \xi_{n}$ converge to those of $\xi$;

b) the sequence $\left(v_{n}^{-1} \xi_{n}\right)_{n \geq 1}$ is tight in $\mathrm{H}_{\rho}^{o}(B)$.

The convergence of "finite dimensional distributions" in a) means namely

$$
v_{n}^{-1}\left(\xi_{n}\left(s_{1}\right), \ldots, \xi_{n}\left(s_{m}\right)\right) \underset{n \rightarrow \infty}{\stackrel{B^{m}}{\longrightarrow}}\left(\xi\left(s_{1}\right), \ldots, \xi\left(s_{m}\right)\right)
$$

for $m \geq 1$ and $0 \leq s_{1}<\cdots<s_{m} \leq 1$. This terminology is used here as a convenient analogy with the classical case $B=\mathbb{R}$. But it should not conceal that in general, problems of infinite dimensional weak convergence may appear already at this stage in connection with some central limit theorem in $B$, involving the geometry of $B$. Of course if $v_{n}^{-1} \xi_{n}$ satisfies already a functional central limit theorem in the space $C(B)$, condition a) is automatically fulfilled. 
Let us discuss now the tightness problem. General conditions implying the tightness of a sequence of random elements in $\mathrm{H}_{\rho}^{o}(B)$ may be found in [14] (Th. 2 and Rem. 1). To translate this result in the setting of partial sums process $\xi_{n}$, write for simplicity

$$
t_{k}=t_{j, k}=k 2^{-j}, \quad k=0,1, \ldots, 2^{j}, \quad j=1,2, \ldots
$$

Then the tightness of $\left(v_{n}^{-1} \xi_{n}\right)_{n \geq 1}$ in $\mathrm{H}_{\rho}^{o}(B)$ is easily seen to hold under the conditions:

i) for every $t \in[0,1],\left(v_{n}^{-1} \xi_{n}(t)\right)_{n \geq 1}$ is tight on $B$;

ii) for every positive $\varepsilon$,

$$
\lim _{J \rightarrow \infty} \limsup _{n \rightarrow \infty} \mathbf{P}\left\{\sup _{j \geq J} \frac{1}{v_{n} \rho\left(2^{-j}\right)} \max _{0 \leq k<2^{j}}\left\|\xi_{n}\left(t_{k+1}\right)-\xi_{n}\left(t_{k}\right)\right\| \geq \varepsilon\right\}=0
$$

The following theorem provides a practical way to reduce the checking of ii). It is worth noticing that nothing is assumed about the dependence structure of $\left(X_{n}\right)_{n \geq 1}$ in its statement. Here and throughout the paper, $\log n$ stands for the logarithm with basis 2 , so that $2^{\log n}=n$.

Theorem 2.1. Let $\xi_{n}$ be the partial sums process built on $\left(X_{k}\right)_{k \geq 0}$, defined by (1.4). Then $\left(v_{n}^{-1} \xi_{n}\right)_{n \geq 1}$ is tight in $\mathrm{H}_{\rho}^{o}(B)$ if:

(1) for every $t \in[0,1],\left(v_{n}^{-1} \xi_{n}(t)\right)_{n \geq 1}$ is tight on $B$;

(2) $\frac{1}{v_{n} \rho(1 / n)} \max _{1 \leq i \leq n}\left\|X_{i}\right\|$ converges in probability to 0 ;

(3) for every positive $\varepsilon$,

$$
\lim _{J \rightarrow \infty} \limsup _{n \rightarrow \infty} \mathbf{P}\left\{\max _{J \leq j \leq \log n} \frac{1}{v_{n} \rho\left(2^{-j}\right)} \max _{0 \leq k<2^{j}}\left\|S_{\left[n t_{k+1}\right]}-S_{\left[n t_{k}\right]}\right\| \geq \varepsilon\right\}=0 .
$$

If the $X_{i}$ 's have identical distribution, then Condition 2 can be replaced by

$$
\forall \varepsilon>0, \quad n \mathbf{P}\left(\left\|X_{1}\right\| \geq \varepsilon v_{n} \rho(1 / n)\right) \underset{n \rightarrow \infty}{\longrightarrow} 0 .
$$

Clearly under identical distribution of the $X_{i}$ 's, (2.1) implies Condition 2. Moreover when (2.1) is sufficient for $\left(v_{n}^{-1} \xi_{n}\right)_{n \geq 1}$ to satisfy the invariance principle in $\mathrm{C}(B)$, then we can drop Condition 1 and concentrate on the verification of (2.1) and Condition 3 to prove the invariance principle in $\mathrm{H}_{\rho}^{o}(B)$.

Proof of Theorem 2.1. We have to check ii). Denote by $P_{0}=P_{0}(J, n)$ the probability appearing in Condition ii). Then $P_{0}$ is bounded by $P_{1}+P_{2}$ where

$$
P_{1}:=\mathbf{P}\left\{\max _{J \leq j \leq \log n} \frac{1}{v_{n} \rho\left(2^{-j}\right)} \max _{0 \leq k<2^{j}}\left\|\xi_{n}\left(t_{k+1}\right)-\xi_{n}\left(t_{k}\right)\right\| \geq \varepsilon\right\}
$$

and

$$
P_{2}:=\mathbf{P}\left\{\sup _{j>\log n} \frac{1}{v_{n} \rho\left(2^{-j}\right)} \max _{0 \leq k<2^{j}}\left\|\xi_{n}\left(t_{k+1}\right)-\xi_{n}\left(t_{k}\right)\right\| \geq \varepsilon\right\} .
$$

Estimation of $P_{2}$. As $j>\log n, t_{k+1}-t_{k}=2^{-j}<1 / n$ and then with $t_{k}$ in say $[l / n,(l+1) / n)$, either $t_{k+1}$ is in $(l / n,(l+1) / n]$ or belongs to $((l+1) / n,(l+2) / n]$, where $1 \leq l \leq n-2$ depends on $k$ and $j$.

In the first case, computing $\xi_{n}\left(t_{k+1}\right)-\xi_{n}\left(t_{k}\right)$ by linear interpolation of $\xi_{n}$ between $\xi_{n}(l / n)$ and $\xi_{n}((l+1) / n)$, we obtain

$$
\left\|\xi_{n}\left(t_{k+1}\right)-\xi_{n}\left(t_{k}\right)\right\|=n\left\|X_{l+1}\right\| 2^{-j} \leq 2^{-j} n \max _{1 \leq i \leq n}\left\|X_{i}\right\| .
$$


If $t_{k}$ and $t_{k+1}$ are in consecutive intervals, then

$$
\begin{aligned}
\left\|\xi_{n}\left(t_{k+1}\right)-\xi_{n}\left(t_{k}\right)\right\| & \leq\left\|\xi_{n}\left(t_{k}\right)-\xi_{n}((l+1) / n)\right\|+\left\|\xi_{n}((l+1) / n)-\xi_{n}\left(t_{k+1}\right)\right\| \\
& \leq\left((l+1) / n-t_{k}+t_{k+1}-(l+1) / n\right) n \max _{1 \leq i \leq n}\left\|X_{i}\right\| \\
& =2^{-j} n \max _{1 \leq i \leq n}\left\|X_{i}\right\| .
\end{aligned}
$$

Recalling that $\theta(t)=t^{1 / 2} \rho(1 / t)$ is ultimately non decreasing, this estimate of $\left\|\xi_{n}\left(t_{k+1}\right)-\xi_{n}\left(t_{k}\right)\right\|$ leads to

$$
\begin{aligned}
P_{2} & \leq \mathbf{P}\left\{\sup _{j>\log n} \frac{1}{v_{n} \rho\left(2^{-j}\right)} n 2^{-j} \max _{1 \leq i \leq n}\left\|X_{i}\right\| \geq \varepsilon\right\} \\
& =\mathbf{P}\left\{\frac{n^{1 / 2}}{v_{n}} \max _{1 \leq i \leq n}\left\|X_{i}\right\| \sup _{j>\log n} \frac{1}{2^{j / 2} \rho\left(2^{-j}\right)}\left(n 2^{-j}\right)^{1 / 2} \geq \varepsilon\right\} \\
& \leq \mathbf{P}\left\{\frac{n^{1 / 2}}{v_{n} \theta(n)} \max _{1 \leq i \leq n}\left\|X_{i}\right\| \geq \varepsilon\right\} \\
& =\mathbf{P}\left\{\frac{1}{v_{n} \rho(1 / n)} \max _{1 \leq i \leq n}\left\|X_{i}\right\| \geq \varepsilon\right\},
\end{aligned}
$$

for $n$ large enough, whence by Condition $2, \lim _{n \rightarrow \infty} P_{2}=0$.

Estimation of $P_{1}$. Let $u_{k}=\left[n t_{k}\right]$. Then $u_{k} \leq n t_{k} \leq 1+u_{k}$ and $1+u_{k} \leq u_{k+1} \leq n t_{k+1} \leq 1+u_{k+1}$. Therefore

$$
\left\|\xi_{n}\left(t_{k+1}\right)-\xi_{n}\left(t_{k}\right)\right\| \leq\left\|\xi_{n}\left(t_{k+1}\right)-S_{u_{k+1}}\right\|+\left\|S_{u_{k+1}}-S_{u_{k}}\right\|+\left\|S_{u_{k}}-\xi_{n}\left(t_{k}\right)\right\|
$$

Since $\left\|S_{u_{k}}-\xi_{n}\left(t_{k}\right)\right\| \leq\left\|X_{1+u_{k}}\right\|$ and $\left\|\xi_{n}\left(t_{k+1}\right)-S_{u_{k+1}}\right\| \leq\left\|X_{1+u_{k+1}}\right\|$ we obtain $P_{1} \leq P_{1,1}+2 P_{1,2}$, where

$$
\begin{aligned}
P_{1,1} & :=\mathbf{P}\left\{\max _{J \leq j \leq \log n} \frac{1}{v_{n} \rho\left(2^{-j}\right)} \max _{1 \leq k \leq 2^{j}}\left\|S_{u_{k+1}}-S_{u_{k}}\right\| \geq \frac{\varepsilon}{2}\right\} \\
P_{1,2} & :=\mathbf{P}\left\{\max _{J \leq j \leq \log n} \frac{1}{v_{n} \rho\left(2^{-j}\right)} \max _{1 \leq i \leq n}\left\|X_{i}\right\| \geq \frac{\varepsilon}{4}\right\} .
\end{aligned}
$$

In $P_{1,2}$, the maximum over $j$ is realized for $j=[\log n]$, so $\lim _{n \rightarrow \infty} P_{1,2}=0$ by Condition 2 .

Gathering all the estimates, we finally obtain

$$
\lim _{J \rightarrow \infty} \limsup _{n \rightarrow \infty} P_{0}=\lim _{J \rightarrow \infty} \limsup _{n \rightarrow \infty} P_{1,1}=0
$$

by Condition 3 .

\subsection{Proof of Theorem $\mathbf{1 . 3}$}

We need to check the convergence of finite dimensional distributions and tightness.

The invariance principle in $C(\mathbb{H})$ is established under (1.6) by Dedecker and Merlevède [2] as a special case of their Theorem 5 (see also in [10] Prop. 17 and the discussion p. 21). From this $C(\mathbb{H})$ invariance principle, we already have the convergence of finite dimensional distributions of $n^{-1 / 2} \xi_{n}$ and Condition 1 of our Theorem 2.1 is satisfied. So it remains to check (2.1) and Condition 3.

First we note that our assumption (1.9) implies via Lemma 3.7 below that for every positive constant $c$,

$$
\lim _{t \rightarrow \infty} t \mathbf{P}\left(\left\|X_{0}\right\| \geq c \theta(t)\right)=0 .
$$


So it remains only to check Condition 3 , that is $\lim _{J \rightarrow \infty} \lim \sup _{n \rightarrow \infty} P_{1}(J, n, \varepsilon)=0$, with

$$
P_{1}(J, n, \varepsilon)=\mathbf{P}\left\{\max _{J \leq j \leq \log n} \frac{1}{n^{1 / 2} \rho\left(2^{-j}\right)} \max _{0 \leq k<2^{j}}\left\|S_{u_{k+1}}-S_{u_{k}}\right\| \geq \varepsilon\right\}
$$

where $u_{k}=\left[n t_{k}\right]=\left[n k 2^{-j}\right]$. It is useful to note here that as $j \leq \log n$,

$$
1 \leq u_{k+1}-u_{k} \leq n 2^{-j}+1 \leq 2 n 2^{-j}, \quad 0 \leq k<2^{j}
$$

Let us fix an arbitrary $\delta>0$ and define

$$
\begin{aligned}
& \widehat{\epsilon}_{l}:=\epsilon_{l} \mathbf{1}\left\{\left\|\epsilon_{l}\right\| \leq \delta \theta(n)\right\}-\mathbf{E} \epsilon_{l} \mathbf{1}\left\{\left\|\epsilon_{l}\right\| \leq \delta \theta(n)\right\}, \\
& \widetilde{\epsilon}_{l}:=\epsilon_{l} \mathbf{1}\left\{\left\|\epsilon_{l}\right\|>\delta \theta(n)\right\}-\mathbf{E} \epsilon_{l} \mathbf{1}\left\{\left\|\epsilon_{l}\right\|>\delta \theta(n)\right\} .
\end{aligned}
$$

Since $\mathbf{E} \epsilon_{l}=0, \epsilon_{l}=\widehat{\epsilon}_{l}+\widetilde{\epsilon_{l}}$ and we have

$$
S_{u_{k+1}}-S_{u_{k}}=\sum_{l=-\infty}^{\infty} b_{k, l} \epsilon_{l}=Z_{j, k}^{(1)}+Z_{j, k}^{(2)}
$$

where

$$
Z_{j, k}^{(1)}=\sum_{l=-\infty}^{\infty} b_{k, l} \widehat{\epsilon}_{l}, \quad Z_{j, k}^{(2)}=\sum_{l=-\infty}^{\infty} b_{k, l} \widetilde{\epsilon}_{l}
$$

and

$$
b_{k, l}:=\sum_{i=u_{k}+1}^{u_{k+1}} a_{i-l}
$$

Hence, we have

$$
P_{1}(J, n, \varepsilon) \leq P_{1}^{(1)}(J, n, \varepsilon, \delta)+P_{1}^{(2)}(J, n, \varepsilon, \delta)
$$

where for $i=1,2$,

$$
P_{1}^{(i)}(J, n, \varepsilon, \delta):=P\left\{\max _{J \leq j \leq \log n} \frac{1}{\rho\left(2^{-j}\right)} \max _{0 \leq k<2^{j}}\left\|Z_{j, k}^{(i)}\right\|>\frac{\varepsilon}{2} n^{1 / 2}\right\}
$$

Estimation of $P_{1}^{(2)}(J, n, \varepsilon, \delta)$. First we apply Chebyshev inequality to obtain

$$
P_{1}^{(2)}(J, n, \varepsilon, \delta) \leq \sum_{J \leq j \leq \log n} \frac{4}{\varepsilon^{2} n \rho\left(2^{-j}\right)^{2}} \sum_{0 \leq k<2^{j}} \mathbf{E}\left\|Z_{j, k}^{(2)}\right\|^{2}
$$

Next observe that from Lemma 3.1 below, there is some constant $c_{0}$ such that for any positive integer $m$

$$
\sum_{l=-\infty}^{\infty}\left\|\sum_{i=1}^{m} a_{i-l}\right\|^{2} \leq c_{0} m .
$$


In view of the Hilbertian structure of $\mathbb{H}$ and recalling (2.3), we get then

$$
\begin{aligned}
\mathbf{E}\left\|Z_{j, k}^{(2)}\right\|^{2} & =\sum_{l=-\infty}^{\infty} \mathbf{E}\left\|b_{k, l} \widetilde{\epsilon}_{l}\right\|^{2} \\
& \leq \sum_{l=-\infty}^{\infty}\left\|b_{k, l}\right\|^{2} \mathbf{E}\left\|\widetilde{\epsilon}_{l}\right\|^{2} \\
& \leq c_{0}\left(u_{k+1}-u_{k}\right) \mathbf{E}\left\|\widetilde{\epsilon}_{0}\right\|^{2} \\
& \leq 2 n 2^{-j} c_{0} \mathbf{E}\left\|\widetilde{\epsilon}_{0}\right\|^{2} .
\end{aligned}
$$

Going back to (2.9) with the estimates provided by Lemmas 3.2 and 3.3 below, we obtain for $n \geq J \geq J_{0}$,

$$
P_{1}^{(2)}(J, n, \varepsilon, \delta) \leq \frac{K \delta^{2}}{\varepsilon^{2}} \sup _{t \geq n} t \mathbf{P}\left(\left\|\epsilon_{0}\right\| \geq \delta \theta(t)\right)
$$

where the integer $J_{0}$ depends on $\rho$ only while the constant $K$ depends on $\rho$ and on the sequence $\left(a_{i}\right)_{i \geq 0}$. Thus (1.8) gives for every positive $\delta$

$$
\lim _{n \rightarrow \infty} P_{1}^{(2)}(J, n, \varepsilon, \delta)=0 .
$$

Estimation of $P_{1}^{(1)}(J, n, \varepsilon, \delta)$. Using (2.3), we get

$$
\begin{aligned}
P_{1}^{(1)}(J, n, \varepsilon, \delta) & \leq \sum_{J \leq j \leq \log n} \mathbf{P}\left\{\frac{1}{\left(2 n 2^{-j}\right)^{1 / 2}} \max _{0 \leq k<2^{j}}\left\|Z_{j, k}^{(1)}\right\| \geq \frac{\varepsilon}{2 \sqrt{2}} \theta\left(2^{j}\right)\right\} \\
& \leq \sum_{J \leq j \leq \log n} \mathbf{P}\left\{\max _{0 \leq k<2^{j}} \frac{\left\|Z_{j, k}^{(1)}\right\|}{\left(u_{k+1}-u_{k}\right)^{1 / 2}} \geq \frac{\varepsilon}{2 \sqrt{2}} \theta\left(2^{j}\right)\right\} \\
& \leq \sum_{J \leq j \leq \log } \sum_{0 \leq k<2^{j}} \mathbf{P}\left\{\frac{\left\|Z_{j, k}^{(1)}\right\|}{\left(u_{k+1}-u_{k}\right)^{1 / 2}} \geq \frac{\varepsilon}{2 \sqrt{2}} \theta\left(2^{j}\right)\right\} .
\end{aligned}
$$

In order to use an exponential inequality for $Z_{j, k}^{(1)}$, we need an upper bound for some Orlicz norm (see Section 3 for the relevant material). According to Lemma 3.5 below, for $1<\gamma \leq 2$, we have

$$
\left\|Z_{j, k}^{(1)}\right\|_{\psi_{\gamma}} \leq K_{\gamma} \mathbf{E}\left\|Z_{j, k}^{(1)}\right\|+K_{\gamma}^{\prime}\left(\sum_{l \in \mathbb{Z}}\left\|b_{k, l}\right\|^{\gamma^{\prime}}\right)^{1 / \gamma^{\prime}} \frac{\delta \theta(n)}{\ln ^{1 / \gamma}\{\varphi(\delta \theta(n))\}},
$$

where $\frac{1}{\gamma}+\frac{1}{\gamma^{\prime}}=1$ and $b_{k, l}$ is defined by (2.7).

To control the first term in the bound (2.13), we get by independence of the $b_{l} \widehat{\epsilon}$ 's, Hilbertian structure of $\mathbb{H}$ and (2.10),

$$
\begin{aligned}
\mathbf{E}\left\|Z_{j, k}^{(1)}\right\| \leq\left(\mathbf{E}\left\|Z_{j, k}^{(1)}\right\|^{2}\right)^{1 / 2} & =\left(\sum_{l \in \mathbb{Z}} \mathbf{E}\left\|b_{k, l} \widehat{\epsilon_{l}}\right\|^{2}\right)^{1 / 2} \\
& \leq\left(\sum_{l \in \mathbb{Z}}\left\|b_{k, l}\right\|^{2}\right)^{1 / 2}\left(\mathbf{E}\left\|\widehat{\epsilon_{0}}\right\|^{2}\right)^{1 / 2} \\
& \leq 2\left(\mathbf{E}\left\|\epsilon_{0}\right\|^{2}\right)^{1 / 2}\left(c_{0}\left(u_{k+1}-u_{k}\right)\right)^{1 / 2} .
\end{aligned}
$$


Next, as $\gamma^{\prime} \geq 2$, we get with $M:=\sum_{i \in \mathbb{Z}}\left\|a_{i}\right\|$,

$$
\sum_{l \in \mathbb{Z}}\left\|b_{k, l}\right\|^{\gamma^{\prime}} \leq M^{\gamma^{\prime}-2} c_{0}\left(u_{k+1}-u_{k}\right) .
$$

Implanting these estimates into (2.13), we obtain

$$
\left\|\frac{Z_{j, k}^{(1)}}{\left(u_{k+1}-u_{k}\right)^{1 / 2}}\right\|_{\psi_{\gamma}} \leq K^{\prime}\left(1+\left(n 2^{-j}\right)^{1 / 2-1 / \gamma} \frac{\delta \theta(n)}{\ln ^{1 / \gamma} \varphi(\delta \theta(n))}\right),
$$

with a constant $K^{\prime}$ depending on $\gamma$, on $\left(a_{i}\right)_{i \in \mathbb{Z}}$ and on the distribution of $X_{1}$.

From this point, the remainder part of a detailed proof would be an exact reproduction of the corresponding part in the proof of Theorem 8 in [12], pp. 235-237. So we shall content ourselves with providing some explanation on the role of the parameter $\gamma$. Using iii) in the definition of the class $\mathcal{R}$ we can choose some $\beta>1 / 2$ such that $\theta(t) \ln ^{-\beta}(t)$ is ultimately non decreasing. Then we require that $1 / 2<1 / \gamma<\beta$. Then going back to (2.12) with the exponential inequality resulting from (2.14) and (3.10) leads after some work to

$$
P_{1}^{(1)}(J, n, \varepsilon, \delta) \leq \frac{2 \mathrm{e}^{-J}}{1-\mathrm{e}^{-1}}+4 n \exp \left(-c \frac{\ln \varphi(\delta \theta(n))}{\delta^{\gamma}}\right),
$$

for $n \geq J \geq j_{0}$, provided that $\gamma<(1-\alpha)^{-1}$, where $j_{0}$ is defined as in [12], p.237. It is easily seen that there is always a choice of $\beta$ making compatible both conditions imposed on $\gamma$. It is important to note here that neither $j_{0}$, nor the constant $c$ depend on $\delta$. Next for $\delta<1$ and $n$ large enough we have from [12] or Lemma 3.6 below:

$$
\frac{\ln \varphi(\delta \theta(n))}{\delta^{\gamma} \ln n} \geq \delta^{1 / \beta-\gamma}
$$

hence

$$
4 n \exp \left(-c \frac{\ln \varphi(\delta \theta(n))}{\delta^{\gamma}}\right) \leq 4 \exp \left(\left(1-c \delta^{1 / \beta-\gamma}\right) \ln n\right) .
$$

As $1 / \beta<\gamma$, we can finally choose $\delta$ small enough to make $1-c \delta^{1 / \beta-\gamma}$ negative. This leads to

$$
\limsup _{n \rightarrow \infty} P_{1}^{(1)}(J, n, \varepsilon, \delta) \leq \frac{2 \mathrm{e}^{-J}}{1-\mathrm{e}^{-1}} .
$$

Recalling (2.8) and (2.11), the same upper bound holds for $\lim \sup _{n \rightarrow \infty} P_{1}(J, n, \varepsilon)$, so letting $J$ tend to infinity ends the proof.

\section{TOOLS AND AUXILIARY RESUlts}

The following lemma extends with a more elementary proof Lemma 1 in [9].

Lemma 3.1. If the sequence $a=\left(a_{l}\right)_{l \in \mathbb{Z}}$ in the Banach space $(B,\|\|)$ satisfies

$$
\|a\|_{\ell^{1}(B)}=\sum_{l \in \mathbb{Z}}\left\|a_{l}\right\|<\infty
$$

then

$$
Q_{n}^{2}(a):=\frac{1}{n} \sum_{l \in \mathbb{Z}}\left\|\sum_{i=1-l}^{n-l} a_{i}\right\|^{2} \underset{n \rightarrow \infty}{\longrightarrow}\left\|\sum_{i \in \mathbb{Z}} a_{i}\right\|^{2}
$$


Proof. First it is easily seen by combining the triangle inequalities in $B$ and in $\ell^{2}(\mathbb{R})$, that $Q_{n}$ satisfies the triangle inequality in $\ell^{1}(B)$. Next an elementary computation provides

$$
Q_{n}^{2}(a) \leq\|a\|_{\ell^{1}(B)}^{2} .
$$

Both properties enable us to reduce the problem via a classical $3 \varepsilon$ argument in checking the convergence (3.2) for any $a$ in the dense subspace $\ell_{0}^{1}(B)$ of sequences with finite support. If $a \in \ell_{0}^{1}(B)$, then $a_{i}=0$ for $|i|>i_{0}$, so

$$
Q_{n}^{2}(a)=\frac{1}{n} \sum_{l=1-i_{0}}^{l=n+i_{0}}\left\|\sum_{i=1-l}^{n-l} a_{i}\right\|^{2} .
$$

In the above sums, the $n-2 i_{0}$ blocks $\sum_{i=1-l}^{n-l} a_{i}$ indexed by $l=1+i_{0}, \ldots, n-i_{0}$ are complete, i.e. equal to $A=\sum_{|i| \leq i_{0}} a_{i}=\sum_{i \in \mathbb{Z}} a_{i}$. As it remains $4 i_{0}$ incomplete blocks, each bounded in norm by $\|a\|_{\ell^{1}(B)}^{2}$, the convergence (3.2) follows.

Lemma 3.2. There is an integer $J_{0}$ depending only on $\rho(h)=h^{\alpha} L(1 / h)$, such that for $n \geq J \geq J_{0}$,

$$
T_{J, n}:=\sum_{J \leq j \leq \log n} \frac{1}{\rho\left(2^{-j}\right)^{2}} \leq \frac{2}{2^{\alpha}-1} \frac{n}{\theta(n)^{2}} .
$$

Proof. Let us denote by $m$ the integer part of $\log n$ and put $n^{\prime}:=2^{m}$, so that $n / 2<n^{\prime} \leq n$. Recalling that $\theta(t)=t^{1 / 2} \rho(1 / t)$, we have

$$
T_{J, n}=\sum_{j=J}^{m} \frac{2^{j}}{\theta\left(2^{j}\right)^{2}}=\sum_{l=0}^{m-J} \frac{n^{\prime} 2^{-l}}{\theta\left(n^{\prime} 2^{-l}\right)^{2}}=\frac{n^{\prime}}{\theta\left(n^{\prime}\right)^{2}} \sum_{l=0}^{m-J} v_{n, l},
$$

with $v_{n, l}:=2^{-l} \theta\left(n^{\prime}\right)^{2} \theta\left(n^{\prime} 2^{-l}\right)^{-2}$. To estimate the ratio $v_{n, l+1} / v_{n, l}$, we note that

$$
\frac{\theta(2 s)^{2}}{\theta(s)^{2}}=2^{1-2 \alpha} \frac{L(2 s)^{2}}{L(s)^{2}}
$$

As $L$ is slowly varying, there is some $s_{0}$ depending on $L$ and $\alpha$ such that for $s \geq s_{0}, L(2 s)^{2} L(s)^{-2} \leq 2^{\alpha}$, whence

$$
\frac{\theta(2 s)^{2}}{\theta(s)^{2}} \leq 2^{1-\alpha}, \quad s \geq s_{0} .
$$

If $2^{J} \geq 2 s_{0}$, we obtain $v_{n, l+1} / v_{n, l} \leq 2^{-\alpha}$ for $0 \leq l<m-J$, therefore

$$
T_{J, n} \leq \frac{n^{\prime}}{\theta\left(n^{\prime}\right)^{2}} \frac{1}{1-2^{-\alpha}}
$$

Now, let us fix $J_{0}$ large enough such that $2^{J_{0}} \geq 2 s_{0}$ and $\theta$ is non decreasing on $\left[2^{J_{0}}, \infty\right)$. To obtain (3.3), it remains to note that $n / 2<n^{\prime} \leq n$ and that by (3.4), $\theta\left(n^{\prime}\right)^{2} \geq \theta(n / 2)^{2} \geq 2^{\alpha-1} \theta(n)^{2}$.

Lemma 3.3. If $\lim _{t \rightarrow \infty} t \mathbf{P}\left(\left\|\epsilon_{0}\right\| \geq \delta \theta(t)\right)=0$, then the random element $\widetilde{\epsilon_{0}}$ defined by (2.5) satisfies

$$
\mathbf{E}\left\|\widetilde{\epsilon_{0}}\right\|^{2} \leq C \delta^{2} \frac{\theta(n)^{2}}{n} \sup _{t \geq n} t \mathbf{P}\left(\left\|\epsilon_{0}\right\| \geq \delta \theta(t)\right),
$$

where the positive constant $C$ depends only on $\rho$. 
Proof. To get rid of the centering term in $\widetilde{\epsilon_{0}}$, we use successively triangular inequality, $(a+b)^{2} \leq 2 a^{2}+2 b^{2}$ and $(\mathbf{E}\|Y\|)^{2} \leq \mathbf{E}\|Y\|^{2}$ to obtain

Now we note that

$$
\mathbf{E}\left\|\widetilde{\epsilon}_{0}\right\|^{2} \leq 4 \mathbf{E}\left\|\epsilon_{0} \mathbf{1}\left\{\left\|\epsilon_{0}\right\|>\delta \theta(n)\right\}\right\|^{2} .
$$

$$
\begin{aligned}
\mathbf{E}\left\|\epsilon_{0} \mathbf{1}\left\{\left\|\epsilon_{0}\right\|>\delta \theta(n)\right\}\right\|^{2} & =\int_{0}^{\infty} 2 t \mathbf{P}\left(\left\|\epsilon_{0}\right\| \mathbf{1}\left\{\left\|\epsilon_{0}\right\|>\delta \theta(n)\right\}>t\right) \mathrm{d} t \\
& =\delta^{2} \theta(n)^{2} \mathbf{P}\left(\left\|\epsilon_{0}\right\|>\delta \theta(n)\right)+I_{n, \delta}
\end{aligned}
$$

where

The substitution $t=\delta \theta(s)$ gives

$$
I_{n, \delta}:=\int_{\delta \theta(n)}^{\infty} 2 t \mathbf{P}\left(\left\|\epsilon_{0}\right\|>t\right) \mathrm{d} t
$$

$$
\begin{aligned}
I_{n, \delta} & =\delta^{2} \int_{n}^{\infty} \mathbf{P}\left(\left\|\epsilon_{0}\right\|>\delta \theta(s)\right) 2 \theta(s) \theta^{\prime}(s) \mathrm{d} s \\
& \leq \delta^{2} \sup _{u \geq n} u \mathbf{P}\left(\left\|\epsilon_{0}\right\| \geq \delta \theta(u)\right) \int_{n}^{\infty} \frac{2 \theta(s) \theta^{\prime}(s)}{s} \mathrm{~d} s .
\end{aligned}
$$

Integrating by parts and noting that $\theta(s)^{2} / s$ vanishes at infinity, we obtain

$$
\int_{n}^{\infty} \frac{2 \theta(s) \theta^{\prime}(s)}{s} \mathrm{~d} s=-\frac{\theta(n)^{2}}{n}+\int_{n}^{\infty} \frac{\rho(1 / s)^{2}}{s} \mathrm{~d} s \leq \int_{0}^{1 / n} \frac{\rho(u)^{2}}{u} \mathrm{~d} u .
$$

The weight function $\rho$ satisfies (see (8) in [12])

$$
\int_{0}^{h} \frac{\rho(u)}{u} \mathrm{~d} u \leq c_{2} \rho(h), \quad 0<h \leq 1 .
$$

As $\rho$ is non decreasing, this leads to

$$
\int_{n}^{\infty} \frac{2 \theta(s) \theta^{\prime}(s)}{s} \mathrm{~d} s \leq c_{2} \rho(1 / n)^{2}=c_{2} \frac{\theta(n)^{2}}{n} .
$$

Reporting the estimates (3.7) and (3.8) in (3.6) leads to the inequality (3.5) with $C=1+c_{2}$.

Let us give now some hints about the Orlicz norms used in the paper. Set for $\gamma \geq 1$, and $X$ a random element in the Banach space $(B,\|\|)$,

$$
\|X\|_{\psi_{\gamma}}:=\inf \left\{c>0 ; \mathbf{E} \exp \left(\|X / c\|^{\gamma}\right) \leq 2\right\} .
$$

Then $\|X\|_{\psi_{\gamma}}$ defines a norm on the space of random elements in $B$ satisfying $\mathbf{E} \exp \left(\|X / c\|^{\gamma}\right)<\infty$ for some $c$ and it is easily seen that

$$
\mathbf{P}\{\|X\| \geq x\} \leq 2 \exp \left(-\frac{x^{\gamma}}{\|X\|_{\psi_{\gamma}}^{\gamma}}\right), \quad x>0 .
$$

The following result provides an useful bound for the $\psi_{\gamma}$ Orlicz norm of a finite sum of independent random elements in $B$.

Theorem 3.4 (Talagrand [16, Th. 4]). Let $\left(Y_{i}\right)_{i \in \mathbb{N}}$ be a sequence of independent mean zero random elements in the Banach space $(B,\|\|)$. Then for $1<\gamma \leq 2$, and any finite subset I of $\mathbb{N}$,

$$
\left\|\sum_{i \in I} Y_{i}\right\|_{\psi_{\gamma}} \leq K_{\gamma}\left\{\mathbf{E}\left\|\sum_{i \in I} Y_{i}\right\|+\left(\sum_{i \in I}\left\|Y_{i}\right\|_{\psi_{\gamma}}^{\gamma^{\prime}}\right)^{1 / \gamma^{\prime}}\right\},
$$


where $1 / \gamma+1 / \gamma^{\prime}=1$ and $K_{\gamma}$ depends on $\gamma$ only.

Lemma 3.5. Let $Z=\sum_{l=-\infty}^{\infty} b_{l} \widehat{\epsilon}_{l}$ where $\widehat{\epsilon}_{l}$ is defined by (2.4) and the $b_{l}$ 's are bounded linear operators $B \rightarrow \mathbb{H}$, with $\sum_{l \in \mathbb{Z}}\left\|b_{l}\right\|<\infty$. Then the series defining $Z$ converges in $\psi_{\gamma}$ Orlicz norm and

$$
\|Z\|_{\psi_{\gamma}} \leq K_{\gamma} \mathbf{E}\|Z\|+K_{\gamma}^{\prime}\left(\sum_{l \in \mathbb{Z}}\left\|b_{l}\right\|^{\gamma^{\prime}}\right)^{1 / \gamma^{\prime}} \frac{\delta \theta(n)}{\ln ^{1 / \gamma}\{\varphi(\delta \theta(n))\}}
$$

where $\varphi$ is the generalized inverse of $\theta$ defined by (1.2), $K_{\gamma}$ is the same as in Theorem 3.4, $K_{\gamma}^{\prime}$ depends on $\rho$, $\gamma$ and the distribution of $\epsilon_{0}$.

Proof. As bounded random elements, the $\widehat{\epsilon}_{l}$ 's have finite $\psi$ Orlicz norm. Then the convergence in $\psi$ Orlicz norm of the series follows easily from the assumption $\sum_{l \in \mathbb{Z}}\left\|b_{l}\right\|<\infty$ and identical distribution of the $\widehat{\epsilon}_{l}$ 's. Combining this convergence and Theorem 3.4 leads to the bound

$$
\|Z\|_{\psi_{\gamma}} \leq K_{\gamma} \mathbf{E}\left\|Z_{k}\right\|+K_{\gamma}\left(\sum_{l \in \mathbb{Z}}\left\|b_{l}\right\|^{\gamma^{\prime}}\right)^{1 / \gamma^{\prime}}\left\|\widehat{\epsilon}_{0}\right\|_{\psi_{\gamma}}
$$

which gives (3.11), applying Lemma 16 in [12] to estimate $\left\|\widehat{\epsilon_{0}}\right\|_{\psi_{\gamma}}$.

Lemma 3.6. Assume that $\rho$ belongs to the class $\mathcal{R}$. Let $\beta$ satisfying Condition iii) in the definition of $\mathcal{R}$. Then for any $0<\delta \leq 1$, there is a $t_{0} \geq 1$, depending on $\rho, \beta$ and $\delta$ such that

$$
\forall t \geq t_{0}, \quad \frac{\ln \varphi(\delta \theta(t))}{\ln t} \geq \delta^{1 / \beta} .
$$

In fact this lemma extracts one simple trick from the proof of Theorem 8 in [12]. It is stated here independently for writing convenience and further use.

Proof. The Condition iii) in the definition of the class $\mathcal{R}$ provides the representation $\theta(t)=f(t) \ln ^{\beta} t, t>1$, with $f$ ultimately non decreasing. This gives in turn $\varphi(u)=\exp \left(u^{1 / \beta} g(u)\right)$ with $g$ ultimately non increasing. Indeed, putting $u=\theta(t)$ and taking the logarithms in this last formula yields $g(\theta(t))=f(t)^{-1 / \beta}$ where $\theta$ is continuous and ultimately non decreasing. Then we have for $t \geq t_{0}$,

$$
\frac{\ln \varphi(\delta \theta(t))}{\ln t}=\frac{\delta^{1 / \beta} \theta(t)^{1 / \beta} g(\delta \theta(t))}{\ln \varphi(\theta(t))}=\frac{\delta^{1 / \beta} g(\delta \theta(t))}{g(\theta(t))} \geq \delta^{1 / \beta} .
$$

We note that the condition $\delta \leq 1$ was used to exploit the ultimate non increasingness of $g$, which explains why $t_{0}$ depends also on $\delta$.

Lemma 3.7. If $\rho$ belongs to the class $\mathcal{R}$, the following conditions are equivalent:

$$
\forall c>0, \quad \lim _{t \rightarrow \infty} t \mathbf{P}\left\{\left\|X_{0}\right\| \geq c \theta(t)\right\}=0
$$

and

$$
\forall c>0, \quad \lim _{t \rightarrow \infty} t \mathbf{P}\left\{\left\|\epsilon_{0}\right\| \geq c \theta(t)\right\}=0 .
$$

Proof. To prove the sufficiency of (3.13) for (3.12), let us fix a positive $\delta$ to be precised later and define

$$
\begin{aligned}
& \widehat{\epsilon_{i}}:=\epsilon_{i} \mathbf{1}\left\{\left\|\epsilon_{i}\right\| \leq \delta \theta(t)\right\}-\mathbf{E} \epsilon_{i} \mathbf{1}\left\{\left\|\epsilon_{i}\right\| \leq \delta \theta(t)\right\}, \\
& \widetilde{\epsilon}_{i}:=\epsilon_{i} \mathbf{1}\left\{\left\|\epsilon_{i}\right\|>\delta \theta(t)\right\}-\mathbf{E} \epsilon_{i} \mathbf{1}\left\{\left\|\epsilon_{i}\right\|>\delta \theta(t)\right\} .
\end{aligned}
$$


Writing for simplicity

$$
\widehat{X_{0}}:=\sum_{i=0}^{\infty} a_{i} \widehat{\epsilon_{i}}, \quad \widetilde{X_{0}}:=\sum_{i=0}^{\infty} a_{i} \widetilde{\epsilon_{i}}
$$

and noting that $X_{0}$ has the same distribution as $\widehat{X_{0}}+\widetilde{X_{0}}$, we have for any $c>0$ and $t>0$,

$$
t \mathbf{P}\left\{\left\|X_{0}\right\| \geq 2 c \theta(t)\right\} \leq t P_{1}+t P_{2},
$$

where

$$
P_{1}:=\mathbf{P}\left\{\left\|\widehat{X_{0}}\right\| \geq c \theta(t)\right\}, \quad P_{2}:=\mathbf{P}\left\{\left\|\widetilde{X_{0}}\right\| \geq c \theta(t)\right\} .
$$

To control $P_{2}$, we get the following bound from Chebyshev inequality, independence of the $a_{i} \widetilde{\epsilon}_{i}$ 's, Hilbertian structure of $\mathbb{H}$ and Lemma 3.3:

$$
P_{2} \leq \frac{\mathbf{E}\left\|\widetilde{\epsilon_{0}}\right\|^{2}}{c^{2} \theta(t)^{2}} \sum_{i=0}^{\infty}\left\|a_{i}\right\|^{2} \leq \frac{C^{\prime} \delta^{2}}{t} \sup _{s \geq t} \mathbf{P}\left\{\left\|X_{0}\right\| \geq \delta \theta(s)\right\}
$$

where the constant $C^{\prime}$ depends on $\theta, c$ and $\left(a_{i}\right)_{i \geq 0}$. In view of (3.13), this gives

$$
\lim _{t \rightarrow \infty} t P_{2}=0
$$

To control $P_{1}$, applying (3.10) with $1<\gamma<2$, we obtain

$$
P_{1} \leq 2 \exp \left(-\frac{(c \theta(t))^{\gamma}}{\left\|\widehat{X_{0}}\right\|_{\psi_{\gamma}}^{\gamma}}\right) .
$$

Next we bound $\left\|\widehat{X_{0}}\right\|_{\psi_{\gamma}}^{\gamma}$ using Lemma 3.5. Noting that $\mathbf{E}\left\|\widehat{X_{0}}\right\|$ is bounded uniformly in $\delta$ and that $\theta(t)^{\gamma} / \ln (\varphi(\delta \theta(t)))$ tends to infinity with $t$ (see (33) in [12]), we obtain

$$
\left\|\widehat{X_{0}}\right\|_{\psi_{\gamma}}^{\gamma} \leq C^{\prime \prime} \frac{\delta^{\gamma} \theta(t)^{\gamma}}{\ln (\varphi(\delta \theta(t)))},
$$

with a constant $C^{\prime \prime}$ depending on $\theta, c,\left(a_{i}\right)_{i \geq 0}$ and $\gamma$. This leads to

$$
t P_{1} \leq 2 \exp \left(\left(1-\frac{C^{\prime \prime} \ln (\varphi(\delta \theta(t)))}{\delta^{\gamma} \ln t}\right) \ln t\right) \leq 2 \exp \left(\left(1-C^{\prime \prime} \delta^{1 / \beta-\gamma}\right) \ln t\right),
$$

according to Lemma 3.6. Choosing $\gamma$ such that $1 / \beta<\gamma<2$ and $\delta$ small enough to make $1-C^{\prime \prime} \delta^{1 / \beta-\gamma}$ negative gives

$$
\lim _{t \rightarrow \infty} t P_{1}=0 .
$$

Together with (3.14), this establishes the sufficiency of (3.13) for (3.12).

Let us prove the necessity of (3.13). We have

$$
X_{0}=a_{0} \epsilon_{0}+\sum_{i=1}^{\infty} a_{i} \epsilon_{-i}=a_{0} \epsilon_{0}+Z
$$


Now, choose $t_{0}>0$ large enough to have both $\mathbf{P}\left\{\|Z\| \leq c \theta\left(t_{0}\right)\right\} \geq 1 / 2$ and $\theta$ non decreasing on $\left[t_{0}, \infty\right)$. Then for $t \geq t_{0}$,

$$
\begin{aligned}
\mathbf{P}\left\{\left\|X_{0}\right\| \geq c \theta(t)\right\} & \geq \mathbf{P}\left\{\left\|a_{0}\right\|\left\|\epsilon_{0}\right\| \geq c \theta(t)+c \theta\left(t_{0}\right)\right\} \mathbf{P}\left\{\|Z\| \leq c \theta\left(t_{0}\right)\right\} \\
& \geq \frac{1}{2} \mathbf{P}\left\{\left\|\epsilon_{0}\right\| \geq \frac{2 c}{\left\|a_{0}\right\|} \theta(t)\right\}
\end{aligned}
$$

due to independence of $\epsilon_{0}$ and $Z$ and the necessity follows.

\section{REFERENCES}

[1] N.H. Bingham, C.M. Goldie and J.L. Teugels, Regular variation. Encyclopaedia of Mathematics and its Applications. Cambridge University Press (1987).

[2] J. Dedecker and F. Merlevède, The conditional central limit theorem in Hilbert spaces. Stoch. Process. Appl. 108 (2003) 229-262.

[3] J. Dedecker, P. Doukhan, G. Lang, J.R. Leon, S. Louhichi and C. Prieur, Weak Dependence: With Examples and Applications, volume 190 of Lect. Notes Statist. Springer (2007).

[4] D. Hamadouche, Invariance principles in Hölder spaces. Portugal. Math. 57 (2000) 127-151.

[5] M. Juodis, A. Račkauskas and Ch. Suquet, Hölderian functional central limit theorems for linear processes. ALEA Lat. Am. J. Probab. Math. Stat. 5 (2009) 47-64.

[6] J. Kuelbs, The invariance principle for Banach space valued random variables. J. Multiv. Anal. 3 (1973) 161-172.

[7] J. Lamperti, On convergence of stochastic processes. Trans. Amer. Math. Soc. 104 (1962) 430-435.

[8] M. Ledoux and M. Talagrand, Probability in Banach Spaces. Springer-Verlag, Berlin, Heidelberg (1991).

[9] F. Merlevède, M. Peligrad and S. Utev, Sharp conditions for the CLT of linear processes in a Hilbert space. J. Theoret. Probab. 10 (1997) 681-693.

[10] F. Merlevède, M. Peligrad and S. Utev, Recent advances in invariance principles for stationary sequences. Probab. Surveys 3 (2006) $1-36$.

[11] A. Račkauskas and Ch. Suquet, Hölder versions of Banach spaces valued random fields. Georgian Math. J. 8 (2001) 347-362.

[12] A. Račkauskas and Ch. Suquet, Necessary and sufficient condition for the Hölderian functional central limit theorem. $J$. Theoret. Probab. 17 (2004) 221-243.

[13] A. Račkauskas and Ch. Suquet, Hölder norm test statistics for epidemic change. J. Statist. Plann. Inference 126 (2004) 495-520.

[14] A. Račkauskas and Ch. Suquet, Central limit theorems in Hölder topologies for Banach space valued random fields. Theor. Probab. Appl. 49 (2004) 109-125.

[15] A. Račkauskas and Ch. Suquet, Testing epidemic changes of infinite dimensional parameters. Stat. Inference Stoch. Process. 9 (2006) 111-134.

[16] M. Talagrand, Isoperimetry and integrability of the sum of independent Banach-space valued random variables. Ann. Probab. 17 (1989) 1546-1570. 